\title{
A review on bioinformatics enrichment analysis tools towards functional analysis of high throughput gene set data
}

\begin{abstract}
The emergence of high throughput data in genomic, proteomic and bioinformatics has increased the necessity to develop large amount of functional analysis tools to facilitate the inference of biologically meaningful data. A promising high throughput strategy, which is the gene-annotation enrichment analysis, can increase the elucidation ability of related biological processes. This paper reviews 35 bioinformatics enrichment tools and 5 gene set databases that are currently available in the field, which include the description of these tools and databases. This information can help tool developers and users to gain a broad view and better understanding on the bioinformatics enrichment tools and databases, enabling a better decision making in choosing tools in particular research interest.
\end{abstract}

Keywords: Bioinformatics; Database; Enrichment tools; Functional analysis; Gene set data; Software 